\title{
On the Dynamics of Abstract Retarded Evolution Equations
}

\author{
Desheng Li, ${ }^{1}$ Jinying Wei, ${ }^{2,3}$ and Jintao Wang ${ }^{1}$ \\ ${ }^{1}$ Department of Mathematics, School of Science, Tianjin University, Tianjin 300072, China \\ ${ }^{2}$ School of Electrical Engineering and Automation, Tianjin University, Tianjin 300072, China \\ ${ }^{3}$ School of Mathematics, Lanzhou City University, Lanzhou 730020, China
}

Correspondence should be addressed to Jinying Wei; weijy2818@163.com

Received 20 July 2013; Accepted 30 August 2013

Academic Editor: Carlo Bianca

Copyright (C) 2013 Desheng Li et al. This is an open access article distributed under the Creative Commons Attribution License, which permits unrestricted use, distribution, and reproduction in any medium, provided the original work is properly cited.

\begin{abstract}
This paper is concerned with the dynamics of the following abstract retarded evolution equation: $(d / d t) u(t)+A u(t)=F(u(t-$ $\left.\left.r_{1}\right), \ldots, u\left(t-r_{n}\right)\right)+g(t)$ in a Hilbert space $H$, where $A: D(A) \subset H \rightarrow H$ is a self-adjoint positive-definite operator with compact resolvent and $F: D\left(A^{\alpha}\right)^{n} \rightarrow H(\alpha \in[0,1 / 2])$ is a locally Lipschitz continuous mapping. The dissipativity and pullback attractors are investigated, and the existence of locally almost periodic solutions is established.
\end{abstract}

\section{Introduction}

This paper is concerned with the abstract retarded evolution equation

$$
\frac{d}{d t} u(t)+A u(t)=F\left(u\left(t-r_{1}\right), \ldots, u\left(t-r_{n}\right)\right)+g(t)
$$

in a Hilbert space $H$, where $A: D(A) \subset H \rightarrow H$ is a selfadjoint positive-definite operator with compact resolvent, $F$ : $D\left(A^{\alpha}\right)^{n} \rightarrow H(\alpha \in[0,1 / 2])$ is a locally Lipschitz continuous mapping with at most a linear growth rate, $g \in C(\mathbb{R} ; H)$ is a bounded function, and $r_{1}, \ldots, r_{n} \geq 0$ are constant time lags. The main purpose here is to study the dynamics of the equation by employing the theory of pullback attractors, LeraySchauder fixed-point theorem, and some basic knowledge on the minimality and recurrence properties in topological dynamics. This consideration is motivated by an increasing interest on the dynamical behavior of retarded evolution equations in recent years.

First, we make a discussion on the dissipativity and existence of pullback attractors of the equation. In the finite dimensional case, Caraballo et al. made a systematic study on such problems for retarded differential equations with and without uniqueness in $[1,2]$ and so forth. The situation in the infinite dimensional case seems to be more complicated. Although there have been a lot of works in this line for some types of retarded partial differential equations and the abstract equations as in (1) (see, e.g., [3-8]), due to some strict restrictions on the nonlinearities, we find that the known results in most of the existing works do not apply to many important PDE examples as the parabolic one given in Section 5 whose nonlinearity involves the gradient $\nabla u$ of the unknown function. In this present work, we will try to establish some new results in more regular spaces under weaker assumptions. In particular, instead of assuming that the corresponding nonlinearities belong to $C\left(H^{n} ; H\right)$ as in the literature, we will assume that $F$ is a mapping from $D\left(A^{\alpha}\right)^{n}$ to $H$ for some $\alpha \in[0,1 / 2]$. It is worth noticing that this allows us to deal with retarded PDEs with more general nonlinearities (see Section 5). Since we are working in a space with more regularities than those in the literature, one has to overcome many extra difficulties in deriving the decay estimates and asymptotic compactness of the solutions.

Then, we are interested in the existence of locally almost periodic solutions. It is well known that an evolution equation with almost periodic external force may fail to have almost periodic solutions (in the Bohr's sense), even in the case where the equation is of a dissipative type. In this paper, we consider a local version of the concept of almost periodicity which will be referred to as the local almost periodicity. We show that the local almost periodicity of a function $h$ is equivalent to the minimality of its hull $\mathscr{H}(h)$ under the Bebutov's dynamical system (with respect to the compactopen topology). With this knowledge, we then prove that if 
$g$ is locally almost periodic, then (1) has at least one locally almost periodic solution.

This paper is organized as follows. In Section 2 we make some preliminary work. In Section 3 we discuss the dissipativity and establish the existence of pullback attractors. In Section 4 we prove the existence of locally almost periodic solutions. Section 5 consists of an example of retarded parabolic equation whose nonlinearity involves the gradient of the unknown function.

\section{Preliminaries}

2.1. Analytic Semigroups. Let $H$ be a Hilbert space with the inner product $(\cdot, \cdot)$ and norm $|\cdot|$. Let

$$
A: D(A) \subset H \longrightarrow H
$$

be a self-adjoint positive-definite operator with compact resolvent, and let

$$
0<\lambda_{1} \leq \lambda_{2} \leq \cdots \leq \lambda_{k} \longrightarrow \infty \quad(k \rightarrow \infty)
$$

be the eigenvalues of $A$ (counting with multiplicity) with the corresponding eigenvectors $\left\{\omega_{i}\right\}_{i=1}^{\infty}$ which form a canonical basis of $H$.

For $\alpha \in \mathbb{R}$, define the powers $A^{\alpha}$ as follows:

$$
A^{\alpha} u=\sum_{i=1}^{\infty} \lambda_{i}^{\alpha} c_{i} \omega_{i}, \quad \forall u:=\sum_{i=1}^{\infty} c_{i} \omega_{i} \in H .
$$

Set

$$
H_{\alpha}:=D\left(A^{\alpha}\right):=\left\{u \in H \mid \sum_{i=1}^{\infty} \lambda_{i}^{2 \alpha} c_{i}^{2}<\infty\right\} .
$$

Then, $H_{\alpha}$ is a Hilbert space. The inner product $(\cdot, \cdot)_{\alpha}$ and norm $|\cdot|_{\alpha}$ of $H_{\alpha}$ are defined, respectively, as

$$
\begin{array}{r}
(u, v)_{\alpha}=\left(A^{\alpha} u, A^{\alpha} v\right), \quad|u|_{\alpha}=(u, u)_{\alpha}^{1 / 2}, \\
\forall u, v \in H_{\alpha} .
\end{array}
$$

It is well known that, for any $-\infty<\alpha<\beta<\infty$, the embedding $H_{\beta} \subset H_{\alpha}$ is compact; moreover, it holds that

$$
|v|_{\alpha}^{2} \leq \lambda_{1}^{2(\alpha-\beta)}|v|_{\beta}^{2}, \quad \forall v \in H_{\beta} .
$$

Denote by $E(t)$ the analytic semigroup generated by $A$ in $H$. The following proposition will play an important role in deriving the decay estimates and can be found in many text books (see, e.g., [9]).

Proposition 1. The following estimates hold true.

(1) Assume that

$$
\|E(t)\| \leq e^{-\lambda_{1} t}, \quad \forall t \geq 0 .
$$

(2) For any $0<\alpha \leq 1$, there exist constants $M_{\alpha}>0$ and $a>0$ such that

$$
\left\|A^{\alpha} E(t)\right\| \leq M_{\alpha} t^{-\alpha} e^{-a t}, \quad \forall t>0 .
$$

(3) For any $0<\alpha \leq 1$, there exists a constant $K_{\alpha}>0$ such that

$$
|(E(t)-I) u| \leq K_{\alpha} t^{\alpha}|u|_{\alpha}, \quad \forall t \geq 0, u \in H_{\alpha} .
$$

2.2. Solutions of the Equation and Its Cauchy Problem. We first give the definition of solutions to (1).

Definition 2. A function $u \in C\left((a-r, b) ; H_{\alpha}\right)$ is said to be a solution of $(1)$ on $(a, b)$, if

(1) $u(t) \in H_{1}=D(A)$ for a.e. $t \in(a, b)$,

(2) $u \in C^{1}(J ; H)$ and solves the equation at a.e. $t \in(a, b)$.

A solution on $\mathbb{R}$ will be called an entire solution.

Denote by $\mathscr{C}$ the space $C\left([-r, 0] ; H_{\alpha}\right)$, which is endowed with the norm $\|\cdot\|_{\mathscr{C}}$ defined as

$$
\|\varphi\|_{\mathscr{C}}=\max _{s \in[-r, 0]}|\varphi(s)|_{\alpha}, \quad \forall \varphi \in \mathscr{C} .
$$

Let $u \in C\left([-r, T) ; H_{\alpha}\right)$. For each $t \in[0, T)$, we will denote by $u_{t}$ the function in $\mathscr{C}$ :

$$
u_{t}(s)=u(t+s), \quad \forall s \in[-r, 0] .
$$

For convenience in statement, the function $u_{t}$ will be referred to as the lifting of $u$ in $\mathscr{C}$.

Given $\varphi \in \mathscr{C}$, consider the Cauchy problem:

$$
\begin{aligned}
\frac{d}{d t} u(t)+A u(t)= & F\left(u\left(t-r_{1}\right), \ldots, u\left(t-r_{n}\right)\right) \\
& +g(t), \quad t>0, \\
& u_{0}=\varphi .
\end{aligned}
$$

Definition 3. A function $u \in C\left([-r, T) ; H_{\alpha}\right)$ is said to be a solution of the Cauchy problem (13), if $u$ is a solution of the equation on $(0, T)$, and, moreover, it fulfills the initial-value condition $u_{0}=\varphi$.

Remark 4. A solution $u$ of the Cauchy problem (13) necessarily satisfies the integral equation:

$$
\begin{aligned}
u(t)= & E(t) \varphi(0) \\
& +\int_{0}^{t} E(t-s)\left(F\left(u\left(s-r_{1}\right), \ldots, u\left(s-r_{n}\right)\right)+g(s)\right) d s .
\end{aligned}
$$

Concerning the existence of solutions for the Cauchy problem, we have the following.

Theorem 5. Suppose that $F: H_{\alpha}^{n} \rightarrow H(0 \leq \alpha<1)$ is a locally Lipschitz continuous mapping, and $g \in C(\mathbb{R} ; H)$.

Then, for any $\varphi \in \mathscr{C}$, the problem (13) has a unique solution $u(t)=u(t ; \varphi)$ on a maximal interval $\left[-r, T_{\varphi}\right)$.

Proof. The proof is quite standard and can be obtained by combining that of Theorem 3.1 in [10], Lemma 47.1 in [9], and Theorem 42.12 in [9]. Here, we give a sketch for completeness and the reader's convenience.

We may assume that $r_{1}=0$. Let $\sigma=\min _{2 \leq k \leq n} r_{k}(>0)$. Define $G_{0}:[0, \sigma] \times H_{\alpha} \rightarrow H$ by

$$
G_{0}(t, v)=F\left(v, \varphi\left(t-r_{2}\right), \ldots, \varphi\left(t-r_{n}\right)\right)+g(t),
$$


and consider the following initial-value problem:

$$
\begin{gathered}
\frac{d}{d t} v(t)+A v(t)=G_{0}(t, v(t)), \quad t \in[0, \sigma] \\
v(0)=\varphi(0) .
\end{gathered}
$$

By the assumptions on $F$ we know that $G_{0}:[0, \sigma] \times H_{\alpha} \rightarrow H$ is a continuous mapping which is locally Lipschitz in $v$. It then follows by [9, Lemma 47.1] that the problem has a unique mild solution:

$$
v_{1} \in C\left(\left[0, \sigma_{1}\right] ; H_{\alpha}\right) \cap L_{\mathrm{loc}}^{2}\left(0, \sigma_{1} ; H_{1}\right)
$$

for some $\sigma_{1} \leq \sigma$. Define

$$
u_{1}(t)= \begin{cases}v_{1}(t), & t \in\left[0, \sigma_{1}\right], \\ \varphi(t), & t \in[-r, 0] .\end{cases}
$$

Then, $u=u_{1}$ is a mild solution of the delay system (13) on $\left[-r, \sigma_{1}\right]$.

Further we can define a mapping $G_{1}:\left[\sigma_{1}, \sigma_{1}+\sigma\right] \times H_{\alpha} \rightarrow$ $H$ by replacing $\varphi$ in (15) with $u_{1}$ and consider the initial value problem:

$$
\begin{gathered}
\frac{d}{d t} v(t)+A v(t)=G_{1}(t, v(t)), \quad t \in\left[\sigma_{1}, \sigma_{1}+\sigma\right], \\
v\left(\sigma_{1}\right)=u_{1}\left(\sigma_{1}\right) .
\end{gathered}
$$

Similar to the above one deduces that this problem has a unique mild solution $v_{2} \in C\left(\left[\sigma_{1}, \sigma_{2}\right] ; H_{\alpha}\right) \cap L_{\text {loc }}^{2}\left(\sigma_{1}, \sigma_{2} ; H_{1}\right)$. Set

$$
u_{2}(t)= \begin{cases}v_{2}(t), & t \in\left[\sigma_{1}, \sigma_{2}\right], \\ u_{1}(t), & t \in\left[-r, \sigma_{1}\right]\end{cases}
$$

One easily verifies that $u_{2}$ is a mild solution of the delay equation (13) on $\left[-r, \sigma_{2}\right]$.

Repeating the above procedure, one can finally obtain a unique mild solution $u \in C\left([-r, T) ; H_{\alpha}\right) \cap L_{\text {loc }}^{2}\left(0, T ; H_{1}\right)$ on some interval $[-r, T)$.

We can also establish a corresponding extension theorem. This can be done as follows. First, suppose that $|u(t)|_{\alpha}$ is bounded as $t \rightarrow T<\infty$. Then, we see that the function

$$
t \longmapsto h(t):=F\left(u\left(t-r_{1}\right), \ldots, u\left(t-r_{n}\right)\right)+g(t)
$$

is bounded in $H$ as $t \rightarrow T$. Therefore, by the basic knowledge on linear equations, it follows that $u(t)$ is continuous in $H_{\alpha}$ at $t=T$. Further, by repeating some argument as above, one can obtain an extension of $u$ on some larger interval $[-r, T+\delta)$. With this fundamental result in hand, we immediately deduce that there exists a maximal interval $\left[-r, T_{\varphi}\right)$ such that the unique solution $u$ of the problem is defined.

This completes the proof of the theorem.

2.3. Dynamical Systems. Now we recall some basic definitions and facts in the theory of nonautonomous dynamical systems on complete metric spaces.
Let $X$ be a complete metric space with the metric $d$. Given any subsets $A$, and $B$ of $X$, define the Hausdorff semidistance $d_{H}(A, B)$ of $A$ and $B$ as

$$
d_{H}(A, B)=\sup _{x \in A} d(x, B)
$$

where $d(x, B)=\min _{y \in B} d(x, y)$.

For any $x \in X$ and $R>0$, we will use $B_{X}(x, R)$ to denote the ball in $X$ centered at $x$ with radius $R$.

A dynamical system $S$ on $X$ is a continuous mapping from $\mathbb{R} \times X$ to $X$ fulfilling the following group properties:

$$
S(0, x)=x, \quad S(t+s, x)=S(s, S(t, x))
$$

for all $t, s \in \mathbb{R}$ and $x \in X$. For notational simplicity we will rewrite $S(t, x)$ as $S_{t} x$.

Let there be given a dynamical system $S$ on $X$. A subset $M \subset X$ is said to be invariant (with respect to $S$ ) if

$$
S_{t} M=M, \quad \forall t \in \mathbb{R} .
$$

A compact invariant set $M$ is said to be minimal if it contains no proper compact invariant subset.

A point $x \in X$ is said to be almost recurrent [11] if, for any $\varepsilon>0$, there exists an $l>0$ such that one can find on any segment of length $l$ a number $\tau$ such that $d\left(S_{\tau} x, x\right)<\varepsilon$.

Denote by $\mathscr{X}$ the space $C_{b}(\mathbb{R} ; X)$ which consists of all the bounded continuous functions from $\mathbb{R}$ to $X$. $\mathscr{X}$ is usually equipped with the metric $\varrho$ which yields the compact-open topology:

$$
\begin{array}{r}
\varrho(u, v)=\sum_{n=1}^{\infty} \frac{1}{2^{n}} \frac{\max _{|t| \leq n} d(u(t), v(t))}{1+\max _{|t| \leq n} d(u(t), v(t))}, \\
\forall u, v \in \mathscr{X} .
\end{array}
$$

For convenience in statement, we will refer to $\varrho$ as the compact-open metric.

Let $\theta=\theta_{t}$ be the translation operator on $\mathscr{X}$ defined by

$$
\theta_{t} u=u(t+\cdot), \quad u \in \mathscr{X} .
$$

Then, $\theta$ is a dynamical system on $\mathscr{X}$, which is usually known as the Bebutov's dynamical system [12].

For a function $u \in \mathscr{X}$, the hull $\mathscr{H}_{\mathscr{X}}(u)$ of $u$ in $\mathscr{X}$ is defined as the closure of the set $\left\{\theta_{t} u \mid t \in \mathbb{R}\right\}$ in $\mathscr{X}$; namely,

$$
\mathscr{H}_{\mathscr{X}}(u)=\overline{\left\{\theta_{t} u \mid t \in \mathbb{R}\right\}} .
$$

\subsection{Pullback Attractors of Cocycles}

Definition 6. Let $X$ and $\Sigma$ be two complete metric spaces, and let there be given a dynamical system $\theta$ on $\Sigma$.

A continuous mapping $\Phi: \mathbb{R}^{+} \times \Sigma \times X \rightarrow X$ is said to be a cocycle on $X$ with the base space $\Sigma$ and driving system $\theta$ if it satisfies the following conditions:

(1) $\Phi(0, h, x)=x$ for any $(h, x) \in \Sigma \times X$;

(2) $\Phi(t+s, h, x)=\Phi\left(t, \theta_{s} h, \Phi(s, h, x)\right)$ for any $t, s \geq 0$ and $(h, x) \in \Sigma \times X$. 
For the sake of simplicity, we will rewrite $\Phi(t, h, x)$ as $\Phi(t, h) x$.

Definition 7. A family $\mathscr{A}=\{A(h)\}_{h \in \Sigma}$ of nonempty compact sets of $X$ is called the global pullback attractor of the cocycle $\Phi$ if, for each $h, A(h)$ is the minimal compact set that enjoys the following pullback attracting property.

For any bounded subset $B$ of $X$,

$$
\lim _{t \rightarrow+\infty} d_{H}\left(\Phi\left(t, \theta_{-t} h\right) B, A(h)\right)=0 .
$$

The following existence result on pullback attractors is well known and can be found in [13-15] and so forth.

Theorem 8. Let $\Phi$ be a cocycle on $X$ with the base space $\Sigma$ and driving system $\theta$. Suppose that there exists a nonempty compact subset $K$ of $X$ such that, for any bounded subset B of $X$,

$$
\Phi(t, h) B \subset K
$$

for $t>0$ sufficiently large.

Then, $\Phi$ has a unique (global) pullback attractor $\mathscr{A}=$ $\{A(h)\}_{h \in \Sigma}$ :

$$
A(h)=\bigcap_{\tau \geq 0} \overline{\bigcup_{t>\tau} \Phi\left(t, \theta_{-t} h\right) K}
$$

\section{Dissipativity of (1)}

In this section, we give a decay estimate and prove the existence of pullback attractors for the Cauchy problem of the equation under appropriate conditions.

From now on we will always assume that $F$ is a locally Lipschitz continuous mapping from $D\left(A^{\alpha}\right)^{n} \rightarrow H$ for some $0 \leq \alpha \leq 1 / 2$, unless otherwise stated.

Let $g \in C_{b}(\mathbb{R} ; H)$ be a bounded function. Denote

$$
g_{0}=\sup _{t \in \mathbb{R}}|g(t)|
$$

3.1. Decay Estimate. The main result in this subsection is contained in the following lemma.

Lemma 9. Suppose that F satisfies the linear growth condition.

(H1) There exist $\beta_{i}>0(1 \leq i \leq n)$ with $\sum_{i=1}^{n} \beta_{i}<\lambda_{1}^{1-\alpha}$ and $k_{1}>0$ such that

$$
\begin{array}{r}
\left|F\left(v_{1}, \ldots, v_{n}\right)\right| \leq \sum_{i=1}^{n} \beta_{i}\left|v_{i}\right|_{\alpha}+k_{1}, \\
\forall\left(v_{1}, \ldots, v_{n}\right) \in H_{\alpha}^{n} .
\end{array}
$$

Then, there exist positive constants $\delta, C_{1}$, and $C_{2}$ depending only on the parameters in (H1) and $g_{0}$ such that, for any solution $u(t)=u(t ; \varphi)$ of $(13)$, one has

$$
|u(t)|_{\alpha}^{2} \leq C_{1}\|\varphi\|_{\mathscr{C}}^{2} e^{-\delta t}+C_{2}, \quad \forall t \geq 0 .
$$

Proof. We fix a $\delta>0$ small enough so that

$$
c_{0}:=\lambda_{1}^{1-\alpha}-\sum_{i=1}^{n} e^{\delta r_{i} / 2} \beta_{i}-\frac{\lambda_{1}^{\alpha}+\lambda_{1}^{-\alpha}}{2} \delta>0 .
$$

For simplicity, denote $l_{i}=\lambda_{1}^{-\alpha} e^{\delta r_{i} / 2}(i=1, \ldots, n)$. Taking the inner product of (1) with $A^{2 \alpha} u$ in $H$, one finds that

$$
\begin{aligned}
& \frac{1}{2} \frac{d}{d t}|u(t)|_{\alpha}^{2}+|u(t)|_{(1 / 2)+\alpha}^{2} \\
& \quad=\left(F\left(u\left(t-r_{1}\right), \ldots, u\left(t-r_{n}\right)\right)+g(t), A^{2 \alpha} u(t)\right) \\
& \quad \leq\left|F\left(u\left(t-r_{1}\right), \ldots, u\left(t-r_{n}\right)\right)+g(t)\right| \cdot|u(t)|_{2 \alpha} .
\end{aligned}
$$

By using (H1) and the Young inequality, we obtain that

$$
\begin{aligned}
& \frac{d}{d t}|u(t)|_{\alpha}^{2}+2|u(t)|_{(1 / 2)+\alpha}^{2} \\
& \quad \leq \sum_{i=1}^{n} \frac{\beta_{i}}{l_{i}}\left|u\left(t-r_{i}\right)\right|_{\alpha}^{2}+\left(\sum_{i=1}^{n} l_{i} \beta_{i}\right)|u(t)|_{2 \alpha}^{2}+\delta|u|_{2 \alpha}^{2}+\frac{k^{2}}{\delta},
\end{aligned}
$$

where $k=k_{1}+g_{0}$. We infer from (7) that

$$
\begin{gathered}
|u(t)|_{2 \alpha}^{2} \leq \lambda_{1}^{2 \alpha-1}|u(t)|_{(1 / 2)+\alpha}^{2}, \\
|u(t)|_{\alpha}^{2} \leq \lambda_{1}^{-1}|u(t)|_{(1 / 2)+\alpha}^{2} .
\end{gathered}
$$

Further by (36) one easily deduces that

$$
\frac{d}{d t}|u(t)|_{\alpha}^{2}+c_{1}|u(t)|_{\alpha}^{2} \leq \sum_{i=1}^{n} \frac{\beta_{i}}{l_{i}}\left|u\left(t-r_{i}\right)\right|_{\alpha}^{2}+\frac{k^{2}}{\delta},
$$

where $c_{1}=2 \lambda_{1}-\sum_{i=1}^{n} \beta_{i} l_{i} \lambda_{1}^{2 \alpha}-\delta \lambda_{1}^{2 \alpha}$.

We rewrite (38) as

$$
\begin{aligned}
& \frac{d}{d t}|u(t)|_{\alpha}^{2}+\delta|u(t)|_{\alpha}^{2} \\
& \quad \leq-\left(c_{1}-\delta\right)|u(t)|_{\alpha}^{2}+\sum_{i=1}^{n} \frac{\beta_{i}}{l_{i}}\left|u\left(t-r_{i}\right)\right|_{\alpha}^{2}+\frac{k^{2}}{\delta} .
\end{aligned}
$$

Multiplying (39) with $e^{\delta t}$ and integrating from 0 to $t$, it yields

$$
\begin{aligned}
|u(t)|_{\alpha}^{2} \leq & e^{-\delta t}|\varphi(0)|_{\alpha}^{2} \\
& -\left(c_{1}-\delta\right) \int_{0}^{t}|u(s)|_{\alpha}^{2} e^{-\delta(t-s)} d s \\
& +\sum_{i=1}^{n} \frac{\beta_{i}}{l_{i}} \int_{0}^{t}\left|u\left(s-r_{i}\right)\right|_{\alpha}^{2} e^{-\delta(t-s)} d s+\frac{k^{2}}{\delta^{2}} .
\end{aligned}
$$

We observe that

$$
\begin{aligned}
\int_{0}^{t}\left|u\left(s-r_{i}\right)\right|_{\alpha}^{2} e^{-\delta(t-s)} d s= & \int_{-r_{i}}^{t-r_{i}}|u(s)|_{\alpha}^{2} e^{-\delta\left(t-s-r_{i}\right)} d s \\
\leq & e^{\delta r_{i}} \int_{0}^{t}|u(s)|_{\alpha}^{2} e^{-\delta(t-s)} d s \\
& +e^{\delta r_{i}} \int_{-r_{i}}^{0}|u(s)|_{\alpha}^{2} e^{-\delta(t-s)} d s .
\end{aligned}
$$


Therefore, by (40), it holds that

$$
\begin{aligned}
|u(t)|_{\alpha}^{2} \leq & e^{-\delta t}|\varphi(0)|_{\alpha}^{2}-c_{2} \int_{0}^{t}|u(s)|_{\alpha}^{2} e^{-\delta(t-s)} d s \\
& +\sum_{i=1}^{n} \frac{\beta_{i}}{l_{i}} e^{\delta r_{i}} \int_{-r_{i}}^{0}|u(s)|_{\alpha}^{2} e^{-\delta(t-s)} d s+\frac{k^{2}}{\delta^{2}} \\
\leq & e^{-\delta t}|\varphi(0)|_{\alpha}^{2} \\
& +\sum_{i=1}^{n} \frac{\beta_{i}}{l_{i}} e^{\delta r_{i}} \int_{-r_{i}}^{0}|u(s)|_{\alpha}^{2} e^{-\delta(t-s)} d s+\frac{k^{2}}{\delta^{2}},
\end{aligned}
$$

where $c_{2}=2 \lambda_{1}^{\alpha} c_{0}$, from which one immediately concludes that

$$
|u(t)|_{\alpha}^{2} \leq\left(1+\sum_{i=1}^{n} \frac{1}{\delta} \beta_{i} \lambda_{1}^{\alpha} e^{\delta r_{i} / 2}\right)\|\varphi\|_{\mathscr{C}}^{2} e^{-\delta t}+\frac{K^{2}}{\delta^{2}} .
$$

This completes the proof of the lemma.

3.2. Existence of Pullback Attractor. Denote by $\mathscr{X}_{\alpha}$ the space $C_{b}\left(\mathbb{R} ; H_{\alpha}\right)$ which is equipped with the compact-open metric $\varrho$. Let $\theta=\theta_{t}$ be the translation operator on $\mathscr{X}_{\alpha}$.

Set $\Sigma=\mathscr{H}_{\mathscr{X}_{0}}(g)$, where $g \in \mathscr{X}_{0}:=C_{b}(\mathbb{R} ; H)$ is the function in (1). For each $(h, \varphi) \in \Sigma \times \mathscr{C}$, consider the Cauchy problem:

$$
\begin{gathered}
\frac{d}{d t} u(t)+A u(t)=F\left(u\left(t-r_{1}\right), \ldots, u\left(t-r_{n}\right)\right)+h(t), \\
t>0, \\
u_{0}=\varphi .
\end{gathered}
$$

By Theorem 5 we know that, under the hypotheses of Lemma 9, (44) has a unique global solution $u(t)=u(t ; h, \varphi)$. Moreover, using some standard argument, it can be easily shown that $u(t ; h, \varphi)$ is continuous in $(t, h, \varphi)$.

Define a continuous mapping $\mathbb{R}^{+} \times \Sigma \times \mathscr{C} \rightarrow \mathscr{C}$ as follows:

$$
\Phi(t, h) \varphi=u_{t}, \quad \forall(t, h, \varphi) \in \mathbb{R}^{+} \times \Sigma \times \mathscr{C},
$$

where $u_{t}$ is the lifting of the solution $u(t)=u(t ; h, \varphi)$ of $(44)$ in $\mathscr{C}$. Then, $\Phi$ is a cocycle on $\mathscr{C}$ with the base space $\Sigma$ and driving system $\theta$.

Lemma 10. Assume the hypotheses in Lemma 9. Then, there exists a bounded uniformly (with respect to $h \in \Sigma$ ) absorbing set $\mathcal{U} \subset \mathscr{C}$ for the system $\Phi$.

Proof. It is clear that the estimate in (33) holds true for solutions of the system (44).

Let $\rho_{\alpha}^{2}=1+C_{2}$, where $C_{2}$ is the positive number in (33). Given any bounded set $D$ in $\mathscr{C}$, by (33) we deduce that there exists a $t_{0}=t_{0}(D)>r>0$ such that

$$
|u(t ; h, \varphi)|_{\alpha} \leq \rho_{\alpha}, \quad \forall t>t_{0}
$$

for all $\varphi \in D$ and $h \in \Sigma$. It follows that $\mathcal{U}=B_{\mathscr{C}}\left(0, \rho_{\alpha}\right)$ is a uniformly absorbing set as we desired.
Remark 11. By using the smoothing property of the operator $A$, it can be easily shown that the set

$$
\left\{u(t ; h, \varphi) \mid t>t_{0}, \varphi \in D, h \in \Sigma\right\}
$$

is contained in a compact subset of $H_{\alpha}$.

Now we state and prove the existence result on pullback attractors.

Theorem 12. Assume the hypotheses in Lemma 9. Then $\Phi$ has a unique global pullback attractor $\mathscr{A}=\{A(h)\}_{h \in \Sigma}$.

Proof. By virtue of Theorem 8, it suffices to show that $\Phi$ has a compact uniformly (with respect $h \in \Sigma$ ) absorbing set.

Let

$$
\begin{gathered}
M=\bigcup_{h \in \Sigma}\left(\bigcup_{t \geq t_{0}(\mathcal{U})+r} \Phi(t, h) \mathcal{U}\right), \\
N=\bigcup_{h \in \Sigma}\left(\bigcup_{t \geq t_{0}(\mathcal{U})+2 r+1} \Phi(t, h) \mathcal{U}\right),
\end{gathered}
$$

where $t_{0}:=t_{0}(\mathcal{U})$ is chosen such that (46) holds for $\mathcal{U}$. Then, we infer from Lemma 10 that

$$
N \subset M \subset \mathcal{U}:=B_{\mathscr{C}}\left(0, \rho_{\alpha}\right) \text {; }
$$

moreover, $N$ is a uniformly (with respect $h \in \Sigma$ ) absorbing set in $\mathscr{C}$.

To complete the proof of the theorem, there remains to check that $N$ is relatively compact in $\mathscr{C}$. For this purpose, by Remark 11 and the classical Ascoli-Arzela theorem, one only needs to verify that $N$ consists of a family of equicontinuous functions.

For any $\psi \in N$, by definition it can be easily seen that there exist $h \in \Sigma$ and $\varphi \in M$ such that

$$
\psi=\Phi(r+1, h) \varphi=u_{r+1},
$$

where $u_{t}$ is the lifting of the solution $u(t)=u(t ; h, \varphi)$ of $(44)$. Note that if $s \in[-r, 0]$, then $r+1+s \in[1, r+1]$. Therefore, to prove the equicontinuity of $N$, we need to check that there exist $L, \gamma>0$ independent of $h \in \Sigma$ and $\varphi \in M$ such that

$$
\left|u\left(t^{\prime}\right)-u(t)\right|_{\alpha} \leq L\left|t^{\prime}-t\right|^{\gamma}, \quad 1 \leq t \leq t^{\prime} \leq r+1 .
$$

For convenience, we rewrite $t^{\prime}$ as $t+\delta$. Then, by (14) we have

$$
|u(t+\delta)-u(t)|_{\alpha} \leq J_{1}+J_{2}+J_{3}
$$

where

$$
\begin{gathered}
J_{1}=|(E(\delta)-I)(E(t) \varphi(0))|_{\alpha}, \\
J_{2}=\int_{0}^{t}|(E(\delta)-I) E(t-s) G(s)|_{\alpha} d s, \\
J_{3}=\int_{t}^{t+\delta}|E(t+\delta-s) G(s)|_{\alpha} d s, \\
G(s)=F\left(u\left(s-r_{1}\right), \ldots, u\left(s-r_{n}\right)\right)+h(s) .
\end{gathered}
$$


Since $\|\varphi\|_{\mathscr{C}} \leq \rho_{\alpha}$, by Lemma 9 we see that

$$
|u(s)|_{\alpha}^{2} \leq\left(C_{1}+1\right) \rho_{\alpha}^{2}, \quad \forall s \geq-r .
$$

Hence one has

$$
\begin{aligned}
|G(s)| & \leq \sum_{i=1}^{n} \beta_{i}\left|u\left(s-r_{i}\right)\right|_{\alpha}+k_{1}+|h(s)| \\
& \leq\left(\sum_{i=1}^{n} \beta_{i}\right) \sqrt{C_{1}+1} \rho_{\alpha}+K_{1}+g_{0}:=C_{\alpha}
\end{aligned}
$$

for all $s \geq 0$, where $g_{0}=\sup _{s \in \mathbb{R}}|g(s)|$. We fix a $0<\gamma<1 / 2$. Then, by using (9) and (10) we deduce, recalling that $t \geq 1$,

$$
\begin{aligned}
J_{1} & \leq\left|(E(\delta)-I)\left(A^{\alpha} E(t) \varphi(0)\right)\right| \\
& \leq K_{\gamma} \delta^{\gamma}\left|A^{\gamma+\alpha} E(t) \varphi(0)\right| \\
& \leq K_{\gamma} \delta^{\gamma} M_{\gamma} t^{-\gamma} e^{-a t}|\varphi(0)|_{\alpha} \\
& \leq K_{\gamma} \delta^{\gamma} M_{\gamma} t^{-\gamma} e^{-a t} \rho_{\alpha} \\
& \leq C_{3} \delta^{\gamma}, \\
J_{2} & \leq \int_{0}^{t}\left|(E(\delta)-I) A^{\alpha} E(t-s) G(s)\right| d s \\
& \leq \int_{0}^{t} K_{\gamma} \delta^{\gamma}\left|A^{\gamma+\alpha} E(t-s) G(s)\right| d s \\
& \leq K_{\gamma} \delta^{\gamma} M_{\alpha+\gamma} C_{\alpha} \int_{0}^{t}(t-s)^{-(\gamma+\alpha)} e^{-a(t-s)} d s \\
& \leq C_{4} \delta^{\gamma}, \\
& \leq C_{\alpha} M_{\alpha} \int_{0}^{\delta}(\delta-s)^{-\alpha} e^{-a(\delta-s)} d s \\
& \leq C_{\alpha}^{t+\delta}\left|A_{t}^{\alpha} E(t+\delta-s) G(s)\right| d s \\
J_{3} & \int_{t}^{t+\delta} M_{\alpha}(t+\delta-s)^{-\alpha} e^{-a(t+\delta-s)} d s \\
& \\
& \\
&
\end{aligned}
$$

Now by (52) one concludes that

$$
\begin{aligned}
\left|u\left(t^{\prime}\right)-u(t)\right|_{\alpha} & =|u(t+\delta)-u(t)|_{\alpha} \\
& \leq\left(C_{3}+C_{4}+C_{5}\right) \delta^{\gamma} \\
& =L\left|t^{\prime}-t\right|^{\gamma} .
\end{aligned}
$$

Note that both $L$ and $\gamma$ are independent of $h \in \Sigma$ and $\varphi \in$ M.

\section{Locally Almost Periodic Solutions}

In general we know that a system with almost periodic forcing term may have no almost periodic solutions even if the system is dissipative. Here, we consider a local version of the concept of almost periodicity in the sense of Bohr. Namely, we introduce a concept of local almost periodicity and prove the existence of locally almost periodic solutions for (1).

We first make a general discussion on locally almost periodic functions.

4.1. Locally Almost Periodic Functions. Let $X$ be a complete metric space with metric $d$, and let $\mathscr{X}$ be the space $C_{b}(\mathbb{R} ; X)$ which is always equipped with the compact-open metric $\varrho$. Denote by $\theta$ the translation operator on $\mathscr{X}$.

Definition 13. A function $u \in \mathscr{X}$ is said to be locally almost periodic if, for any $\varepsilon, T>0$, there exists an $l>0$ such that, for every $s \in \mathbb{R}$, one can find on any segment of length $l$ a number $\tau=\tau(s)$ such that

$$
\max _{|t| \leq T} d\left(\theta_{s} u(t), \theta_{\tau} u(t)\right)<\varepsilon
$$

One easily verifies the validity of the following easy proposition, which actually gives another equivalent definition for locally almost periodic functions.

Proposition 14. $u \in \mathscr{X}$ is locally almost periodic If, for every $\varepsilon>0$, there exists an $l>0$ such that, for every $s \in \mathbb{R}$, one can find on any segment of length $l$ a number $\tau=\tau(s)$ such that

$$
\varrho\left(\theta_{s} u, \theta_{\tau} u\right)<\varepsilon
$$

Proposition 15. If $u$ is a locally almost periodic function, then the following affirmations hold:

(1) $u$ is uniformly continuous on $\mathbb{R}$;

(2) $U:=\overline{\{u(t) \mid t \in \mathbb{R}\}}$ is compact in $X$.

Proof. (1) Let $\varepsilon>0$ be given arbitrarily. We need to prove that there exists a $0<\delta<1$ such that

$$
d\left(u\left(s_{1}\right), u\left(s_{2}\right)\right)<\varepsilon
$$

for all $s_{1}, s_{2} \in \mathbb{R}$ with $\left|s_{1}-s_{2}\right|<\delta$.

By local almost periodicity of $u$, there exists an $l>0$ such that, for any $s \in \mathbb{R}$, there exists a $\tau=\tau(s) \in[0, l]$ such that

$$
d(u(s+t), u(\tau+t))<\frac{\varepsilon}{3}, \quad \forall t \in[-1,1] .
$$

Since $u$ is uniformly continuous on the interval $[-1,1]$, one can find a positive number $\delta<1$ such that

$$
d(u(s), u(t))<\frac{\varepsilon}{3}, \quad \forall s, t \in[-1, l+1],|s-t|<\delta .
$$

We show that $\delta$ satisfies $(60)$.

Indeed, for any $s_{1}, s_{2} \in \mathbb{R}$ with $\left|s_{1}-s_{2}\right|<\delta$, we can pick an $s \in \mathbb{R}$ such that $s_{1}, s_{2} \in[s-1, s+1]$. We may write $s_{1}$ and $s_{2}$ as

$$
s_{1}=s+t_{1}, \quad s_{2}=s+t_{2} .
$$

Then, by (61), there exists a $\tau \in[0, l]$ such that

$$
d\left(u\left(s+t_{i}\right), u\left(\tau+t_{i}\right)\right)<\frac{\varepsilon}{3}, \quad i=1,2 .
$$


Now we have that

$$
\begin{aligned}
d\left(u\left(s_{1}\right), u\left(s_{2}\right)\right)= & d\left(u\left(s+t_{1}\right), u\left(s+t_{2}\right)\right) \\
\leq & d\left(u\left(s+t_{1}\right), u\left(\tau+t_{1}\right)\right) \\
& +d\left(u\left(s+t_{2}\right), u\left(\tau+t_{2}\right)\right) \\
& +d\left(u\left(\tau+t_{1}\right), u\left(\tau+t_{2}\right)\right) \\
\leq & \frac{\varepsilon}{3}+\frac{\varepsilon}{3}+\frac{\varepsilon}{3}=\varepsilon .
\end{aligned}
$$

(2) To prove the compactness of $U$, we employ the Kuratowski's measure $\beta$ of noncompactness on $X$ which is defined as follows: for any $K \subset X$,

$$
\begin{aligned}
\beta(K)=\{\inf \delta>0 \mid & \text { there exist a finite number of } \\
& \text { balls } B_{1}, \ldots, B_{n} \text { in } X \text { with radius } \\
& \left.\delta \text { such that } K \subset \bigcup_{1 \leq i \leq n} B_{i}\right\} .
\end{aligned}
$$

It is well known that $K$ is precompact if and only if $\beta(K)=0$.

In what follows we show that $\beta(U)=0$, thus proving what we desired.

Let $\varepsilon>0$ be given arbitrarily. Then, by local almost periodicity of $u$, there exists an $l>0$ such that, for any $s \in \mathbb{R}$, one can find on any segment of length $l$ a number $\tau=\tau(s)$ such that

$$
\max _{|t| \leq 1} d\left(\theta_{s} u(t), \theta_{\tau} u(t)\right)<\varepsilon
$$

In particular, we see that, for any $s \in \mathbb{R}$, there exists a $\tau \in I:=$ $[0, l]$ such that

$$
d(u(s), u(\tau))<\varepsilon .
$$

It then follows that

$$
U \subset\{x \in X \mid d(x, u(I))<\varepsilon\},
$$

where $u(I)=\{u(t) \mid t \in I\}$. Since $u(I)$ is compact, one easily deduces by (69) that $\beta(U) \leq 2 \varepsilon$. Hence, $\beta(U)=0$. The proof is complete.

The following result shows that the local almost periodicity of a function is actually equivalent to the minimality of the hull of the function under the Bebutov's dynamical system and is of crucial importance in proving the existence of locally almost periodic solutions.

Theorem 16. A function $u \in \mathscr{X}$ is locally almost periodic if and only if $\mathscr{H}_{\mathscr{X}}(u)$ is minimal under the Bebutov's dynamical system.

Proof. “ $\models$ " Assume that $\mathscr{H}_{X}(u)$ is minimal. Then, by the basic knowledge in the theory of dynamical systems (see, e.g.,
$[11,16])$, we know that every $v \in \mathscr{H}_{X}(u)$ is almost recurren; that is, for any $\varepsilon>0$, there exists an $l>0$ such that one can find on any segment of length $l$ a number $\tau$ such that

$$
\varrho\left(v, \theta_{\tau} v\right)<\varepsilon
$$

By a simple argument via contradiction, one can easily show that the length $l$ of the segments can be independent of $v$ (see also the work of Birkhoff [16]). Thanks to Proposition 14, one immediately concludes by (70) and the independence of $l$ on $v$ that $u$ is locally almost periodic.

" $\Rightarrow$ " Conversely, if $u$ is locally almost periodic, then by Proposition $15 u$ is uniformly continuous on $\mathbb{R}$ with $\{u(t)$ $t \in \mathbb{R}\}$ being precompact in $X$. Therefore, by the classical Ascoli-Arzela theorem, one deduces that, for any sequence $\tau_{n} \in \mathbb{R}$, the sequence $\theta_{\tau_{n}} u$ has a subsequence $\theta_{\tau_{n_{k}}} u$ that converges uniformly on any compact interval $I$ to a function $v \in$ $\mathscr{X}$. Hence, we have

$$
\lim _{k \rightarrow \infty} \varrho\left(\theta_{\tau_{n_{k}}} u, v\right)=0 .
$$

This implies that the hull $\mathscr{H}_{\mathscr{X}}(u)$ is compact.

On the other hand, by the local almost periodicity of $u$ and Proposition 14, we see that $u$ is an almost recurrent point of the system $\theta$. Since $\mathscr{H}_{X}(u)$ is compact, by adopting some argument as in [16], it can be easily shown that $\mathscr{H}_{\mathscr{X}}(u)$ is minimal. We omit the details.

4.2. Existence of Locally Almost Periodic Solutions. Now we consider the existence of locally almost periodic solutions for (1). The main result is contained in the following theorem.

Theorem 17. Suppose that F satisfies (H1). Then, if $g$ is locally almost periodic, (1) has at least one locally almost periodic solution.

Proof. Denote by $\mathscr{X}_{\alpha}$ the space $C_{b}\left(\mathbb{R} ; H_{\alpha}\right)$ which is equipped with the compact-open metric $\varrho$. Denote by $\theta$ the translation operator on $\mathscr{X}_{\alpha}$.

Let $\Phi$ be the cocycle on the phase space $\mathscr{C}:=C([-r, 0]$; $H_{\alpha}$ ) generated by (44) (see (45) for the definition). Define the skew-product flow $\pi: R^{+} \times \Sigma \times \mathscr{C} \rightarrow \Sigma \times \mathscr{C}$ as follows:

$$
\pi(t, h, \varphi):=\left(\theta_{t} h, \Phi(t, h) \varphi\right), \quad \forall(h, \varphi) \in \Sigma \times \mathscr{C}, t \geq 0 .
$$

Then, as in the proof of Theorem 12, one can easily show that $\pi$ has a global attractor $\mathscr{A}$ in $\Sigma \times \mathscr{C}$ which is the maximal compact invariant set that attracts each bounded subset of $\Sigma \times \mathscr{C}$. Further, by a recurrence theorem due to Birkhoff and Bebutov (see, e.g., [12]), $\mathscr{A}$ contains a compact minimal invariant set $\mathscr{M}$ of the system $\pi$.

Fix a $\left(h_{0}, \varphi_{0}\right) \in \mathscr{M}$. Then, by the invariance property of $\mathscr{M}$, there exists a trajectory $\gamma$ of $\pi$ which is defined on the whole line $\mathbb{R}$ and contained in $\mathscr{M}$ such that $\gamma(0)=\left(h_{0}, \varphi_{0}\right)$. Let $\gamma(t)=\left(\theta_{t} h_{0}, v_{t}\right)$. Define a function $v$ as

$$
v(t)=v_{t}(0), \quad t \in \mathbb{R} .
$$

Then, one easily sees that $v$ is an entire solution of the equation in (44) with $h$ therein replaced by $h_{0}$. We also infer 
from the proof of Theorem 12 that $v$ is uniformly continuous on $\mathbb{R}$ with $\{v(t) \mid t \in \mathbb{R}\}$ being contained in a compact subset of $H_{\alpha}$. Therefore by the classical Ascoli-Arzela theorem, the hull $\mathscr{H}_{\mathscr{X}_{\alpha}}(v)$ in $\mathscr{X}_{\alpha}$ is compact.

Let $\mathfrak{J}:=\mathscr{H}_{\mathscr{X}_{0} \times X_{a}}\left(h_{0}, v\right)$. Note that if $(h, w) \in \mathfrak{J}$, then it solves the following equation:

$$
\begin{aligned}
\frac{d}{d t} w(t)+A w(t)= & F\left(w\left(t-r_{1}\right), \ldots, w\left(t-r_{n}\right)\right) \\
& +h(t), \quad t \in R .
\end{aligned}
$$

Observe that

$$
P \mathfrak{I}=\mathscr{H}_{\mathscr{X}_{0}}\left(h_{0}\right)=\Sigma, \quad Q \mathfrak{I}=\mathscr{H}_{\mathscr{X}_{\alpha}}(v),
$$

where $P$ and $Q$ are projection operators from $\mathscr{X}_{0} \times \mathscr{X}_{a}$ to $\mathscr{X}_{0}$ and $\mathscr{X}_{\alpha}$, respectively. Since both $\Sigma$ and $\mathscr{H}_{\mathscr{X}_{\alpha}}(v)$ are compact, we deduce that $\mathfrak{I}$ is compact.

Now we show that $\mathfrak{J}$ is minimal. Suppose the contrary. Then, it would contain a proper compact invariant subset $\mathscr{K}$. Let

$$
\mathscr{K}=\left\{h, w_{0} \mid(h, w) \in \mathscr{K}\right\},
$$

where $w_{0} \in \mathscr{C}$ is the restriction of $w$ on $[-r, 0]$,

$$
w_{0}(s)=w(s), \quad s \in[-r, 0]
$$

It is trivial to check that $\mathscr{K}$ is a proper compact invariant subset of $\mathscr{M}$, which contradicts to the minimality of $\mathscr{M}$.

By the first equation in (75), we see that there exists a $u \in \mathscr{X}_{\alpha}$ such that $(g, u) \in \mathfrak{I}$. Further, by the minimality of $\mathfrak{I}$, one finds that $\mathscr{H}_{\mathscr{X}_{0} \times X_{a}}(g, u)=\mathfrak{J}$. Hence $\mathscr{H}_{\mathscr{X}_{0} \times X_{a}}(g, u)$ is minimal. It then follows immediately by Theorem 16 that $u$ is a locally almost periodic function. By (69), we see that $u$ is precisely an entire solution of (1).

\section{An Example}

We now give an example to demonstrate how the abstract results in previous sections can be applied to nonautonomous parabolic equations with delays. $\mathbb{R}^{N}$ :

Let $L$ be a differential operator on a bounded domain $\Omega \subset$

$$
L u:=-\sum_{i, j=1}^{N} \frac{\partial}{\partial x_{i}}\left(a_{i j}(x) \frac{\partial u}{\partial x_{j}}\right)+a_{0}(x) u
$$

where $a_{i j}, a_{0} \in L^{\infty}(\Omega)$ and $a_{i j}=a_{j i}$ for all $1 \leq i, j \leq N$. We assume that there exists a constant $v>0$ such that, for a.e. $x \in \Omega$,

$$
\sum_{i, j=1}^{N} a_{i j}(x) \xi_{i} \xi_{j} \geq v|\xi|^{2}, \quad \forall \xi=\left(\xi_{1}, \ldots, \xi_{N}\right) \in \mathbb{R}^{N}
$$

and that

$$
a_{0}(x) \geq 0, \quad \text { a.e. } x \in \bar{\Omega} .
$$

Hence, $L$ is uniformly elliptic on $\Omega$.
Let $f: \mathbb{R}^{N+1} \rightarrow \mathbb{R}$ be a locally Lipschitz continuous function. Consider the retarded parabolic equation on $\Omega$ :

$$
\frac{\partial u}{\partial t}+L u=f\left(u\left(x, t-r_{1}\right), \nabla u\left(x, t-r_{2}\right)\right)+g(x, t)
$$

associated with the homogeneous Dirichlet boundary condition:

$$
\left.u\right|_{\partial \Omega}=0
$$

where $r_{1}, r_{2}>0$ denote time lags.

Let $H=L^{2}(\Omega), V=H_{0}^{1}(\Omega)$. Define a symmetric bilinear form $B(u, v)$ on $V$ as follows:

$$
B(u, v)=\int_{\Omega}\left(a_{i j}(x) \frac{\partial u}{\partial x_{j}} \frac{\partial v}{\partial x_{j}}+a_{0}(x) u v\right) d x, \quad u, v \in V
$$

It is clear that $B(u, v)$ is bounded and coercive. Thanks to the Lax-Milgram theorem, $B(u, v)$ generates a self-adjoint positive-definite operator $A$ on $H$ with compact resolvent. Note that

$$
V=D\left(A^{1 / 2}\right):=H_{1 / 2}
$$

We assume that $f$ satisfies the following linear growth condition.

(F1) There exist positive constants $b_{0}, \ldots, b_{N}$ and $k$, such that

$$
|f(z)| \leq \sum_{i=0}^{N} b_{i}\left|z_{i}\right|+k, \quad \forall z=\left(z_{0}, \ldots, z_{N}\right) \in \mathbb{R}^{N+1}
$$

Then, one easily sees that the mapping $F: V^{2} \rightarrow H$ defined by

$$
F(u, v)=f(u, \nabla v), \quad u, v \in V
$$

makes sense and is locally Lipschitz. Setting $g(t)=g(\cdot, t)$, the problem (81)-(82) can be reformulated as an abstract equation in $H$ as follows:

$$
\frac{d u}{d t}+A u=F\left(u\left(t-r_{1}\right), u\left(t-r_{2}\right)\right)+g(t)
$$

Now we are in a situation of (1).

Since $f$ satisfies (F1), then one can easily verify that the mapping $F$ satisfies (H1) in the previous sections, and hence the abstract results obtained therein apply. In particular, we have the following.

Theorem 18. Assume that $f$ satisfies the linear growth condition (F1) with the positive constants $b_{i}$ 's therein satisfying

$$
b_{0} \lambda_{1}^{-1 / 2}+\sum_{i=1}^{N} b_{i}<\lambda_{1}^{1 / 2}
$$

where $\lambda_{1}$ is the first eigenvalue of $A$. Let $g \in C_{b}(\mathbb{R} ; H)$ be a locally almost periodic (resp., periodic) function.

Then, (87) has at least one locally almost periodic (resp., periodic) solution. 


\section{Conflict of Interests}

The authors declare that there is no conflict of interests regarding the publication of this paper.

\section{Acknowledgment}

This paper is supported by the Grant of NSF of China (10771159, 11071185).

\section{References}

[1] T. Caraballo, P. Marín-Rubio, and J. Valero, "Autonomous and non-autonomous attractors for differential equations with delays," Journal of Differential Equations, vol. 208, no. 1, pp. 9-41, 2005.

[2] T. Caraballo, M. J. Garrido-Atienza, B. Schmalfuss, and J. Valero, "Non-autonomous and random attractors for delay random semilinear equations without uniqueness," Discrete and Continuous Dynamical Systems, vol. 21, no. 2, pp. 415-443, 2008.

[3] T. Caraballo and J. Real, "Attractors for 2D-Navier-Stokes models with delays," Journal of Differential Equations, vol. 205, no. 2, pp. 271-297, 2004.

[4] J. Li and J. Huang, "Uniform attractors for non-autonomous parabolic equations with delays," Nonlinear Analysis, Theory, Methods and Applications, vol. 71, no. 5-6, pp. 2194-2209, 2009.

[5] M. A. Ragusa, "Cauchy-Dirichlet problem associated to divergence form parabolic equations," Communications in Contemporary Mathematics, vol. 6, no. 3, pp. 377-393, 2004.

[6] A. V. Rezounenko, "Partial differential equations with discrete and distributed state-dependent delays," Journal of Mathematical Analysis and Applications, vol. 326, no. 2, pp. 1031-1045, 2007.

[7] A. V. Rezounenko, "A condition on delay for differential equations with discrete state-dependent delay," Journal of Mathematical Analysis and Applications, vol. 385, no. 1, pp. 506-516, 2012.

[8] J. W.-H. So and J. H. Wu, "Topological dimensions of global attractors for semilinear PDEs with delays," Bulletin of the Australian Mathematical Society, vol. 43, no. 3, pp. 407-422, 1991.

[9] G. R. Sell and Y. You, "Dynamics of evolutionary equations”, in Applied Mathematical Sciences, vol. 143, Springer, New York, NY, USA, 2002.

[10] Y. X. Li, "Existence and asymptotic stability of periodic solution for evolution equations with delays," Journal of Functional Analysis, vol. 261, no. 5, pp. 1309-1324, 2011.

[11] V. P. Bongolan-Walsh, D. Cheban, and J. Duan, "Recurrent motions in the nonautonomous Navier-Stokes system," Discrete and Continuous Dynamical Systems B, vol. 3, no. 2, pp. 255-262, 2003.

[12] G. R. Sell, "Topological dynamics and ordinary differential equations," in Van Nostrand Reinhold Mathematical Studies, vol. 33, Van Nostrand Reinhold, London, UK, 1971.

[13] D. N. Cheban, P. E. Kloeden, and B. Schmalfuss, "The relationship between pullback, for- wards and global attractors of nonautonomous dynamical systems," Nonlinear Dynamics and Systems Theory, vol. 2, pp. 125-144, 2002.

[14] V. V. Chepyzhov and M. I. Vishik, Attractors for Equations of Mathematical Physics, vol. 49, American Mathematical Society, Providence, RI, USA, 2002.
[15] H. Song and H. Wu, "Pullback attractors of nonautonomous reaction-diffusion equations," Journal of Mathematical Analysis and Applications, vol. 325, no. 2, pp. 1200-1215, 2007.

[16] G. D. Birkhoff, "Dynamical systems," in American Mathematical Society Colloquium, vol. 9, American Mathematical Society, Providence, RI, USA, 1927. 


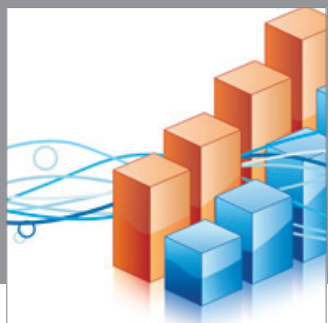

Advances in

Operations Research

mansans

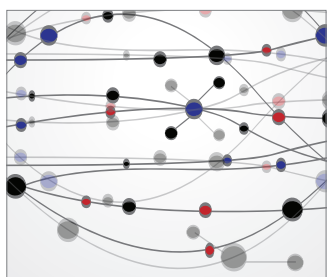

The Scientific World Journal
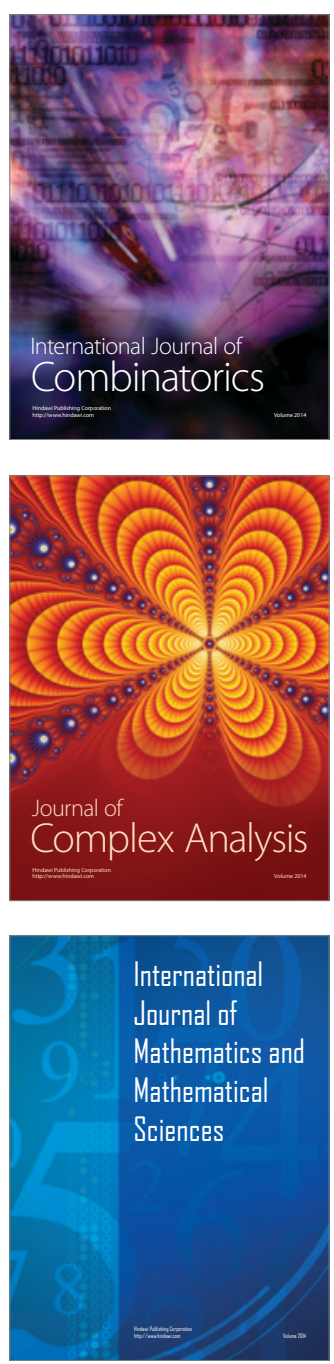
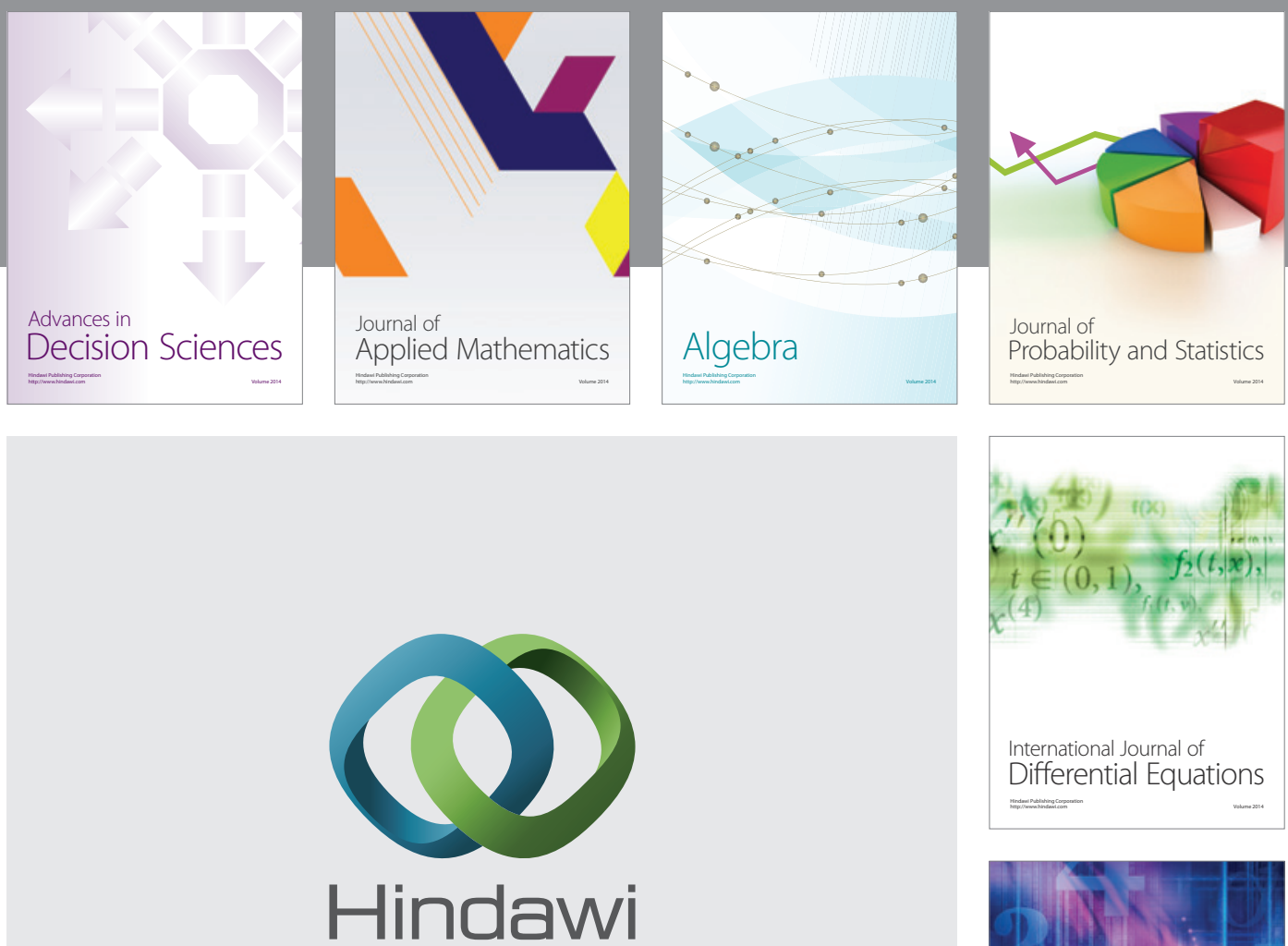

Submit your manuscripts at http://www.hindawi.com
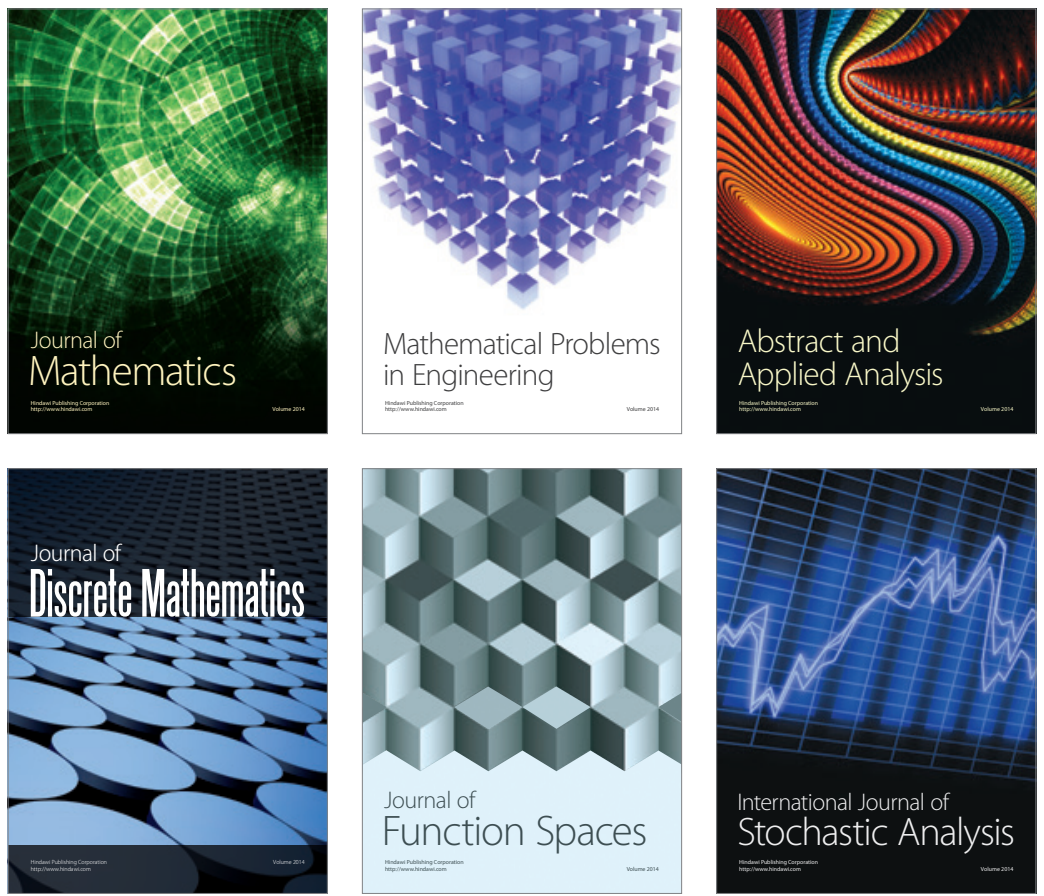

Journal of

Function Spaces

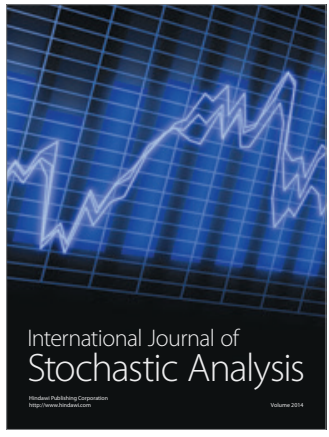

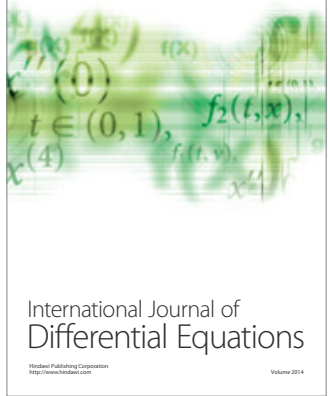
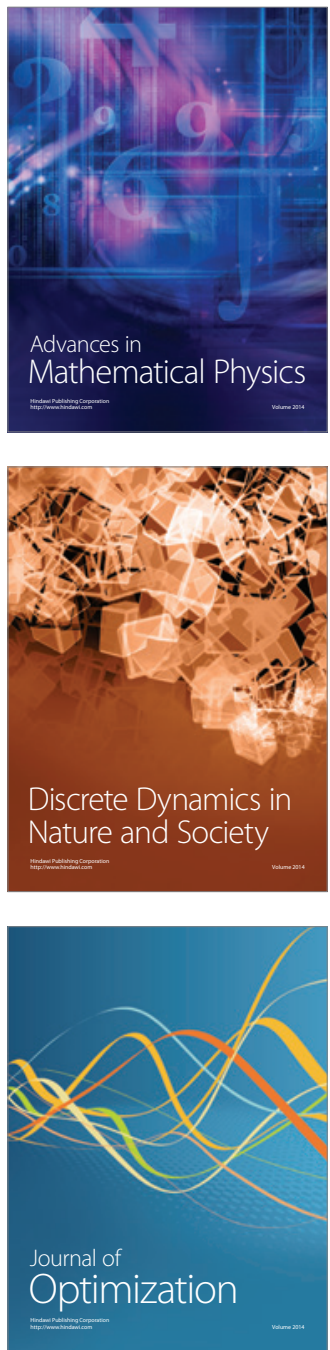